\title{
An Ultra-Low-Power Digitally-Controlled Buck Converter IC for Cellular Phone Applications
}

\author{
Jinwen Xiao \\ Angel Peterchev \\ Jianhui Zhang \\ Seth Sanders \\ University of California, Berkeley \\ Email: xjw, peterch, zhangjh, sanders@wind.eecs.berkeley.edu
}

\begin{abstract}
This paper describes a dual-mode digitallycontrolled buck converter IC for cellular phone applications. An architecture employing internal power management is introduced to ensure voltage compatibility between a singlecell lithium-ion battery voltage and a low voltage integrated circuit technology. Special purpose analog and digital interface elements are developed. These include a ring-oscillator based ADC (ring-ADC), which is nearly entirely synthesizable, robust against switching noise, and has flexible resolution control, and a very low power ring oscillator-multiplexer based Digital PWM generation module (ring-MUX DPWM). The chip, which includes an output power stage rated for $400 \mathrm{~mA}$, occupies $2 \mathrm{~mm}^{2}$ active area in $0.25-\mu \mathrm{m}$ CMOS. Very high efficiencies are achieved over a load range of 0.1 to $400 \mathrm{~mA}$. Measured quiescent current in PFM mode is $4 \mu \mathrm{A}$.
\end{abstract}

\section{INTRODUCTION}

This paper presents an ultra-low-quiescent-power dualmode digitally-controlled buck converter IC for cellular phone applications. While the cellular phone is in talk mode, the load on the buck converter is high and pulse width modulation (PWM) is used to achieve high regulation quality as well as high efficiency. However, when the cellular phone is in standby mode, in which the load current is very low, PWM mode leads to low efficiency due to excessive switching, gate drive, and quiescent current losses. To extend the standby time a cellular phone can sustain with each full charge of the battery, pulse frequency modulation (PFM) mode is preferred for light load operation. The designed IC supports PWM mode for heavy load and PFM mode for light load. The system block diagram of the buck converter IC and the external filter is shown in Fig. 1. The pin MODE is used to switch between the two modes. The PFM mode quiescent power is the fundamental limitation on light-load efficiency, and in this work, an ultra low quiescent current of $4 \mu \mathrm{A}$ is achieved in PFM mode. Details of the dual-mode design are presented in Section II.

For the digital implementation, small feature size processes with low supply voltages are preferred for implementing the digital circuits to achieve small die area, high speed, and low power consumption. In cellular phone applications, the power supply of the buck converter system is usually a single cell lithium-ion battery, with voltage range of 2.8$5.5 \mathrm{~V}$. Thus, the input voltage of the converter may be higher than the allowed supply voltage of the process (e.g., maximum $2.75 \mathrm{~V}$ for a $0.25-\mu \mathrm{m}$ CMOS process). A solution that allows a low voltage process to be used for the digital controller with high input voltage can be of great interest considering the possible cost reduction from integrating the switching regulator on the same die with the power train devices. Internal power management is introduced to resolve the conflict of high input voltage and a low voltage process, the details of which are also presented in Section II.

The Analog-to-Digital Converter (ADC) and the Digital Pulse Width Modulator (DPWM) are used to provide the analog and digital interface between the digital compensation network and the buck converter. A general purpose ADC can be unnecessarily expensive in terms of both power consumption and chip area, therefore a novel ring-oscillatorbased averaging ADC (ring-ADC) is developed which has reduced quantization range. The details of the ADC are 


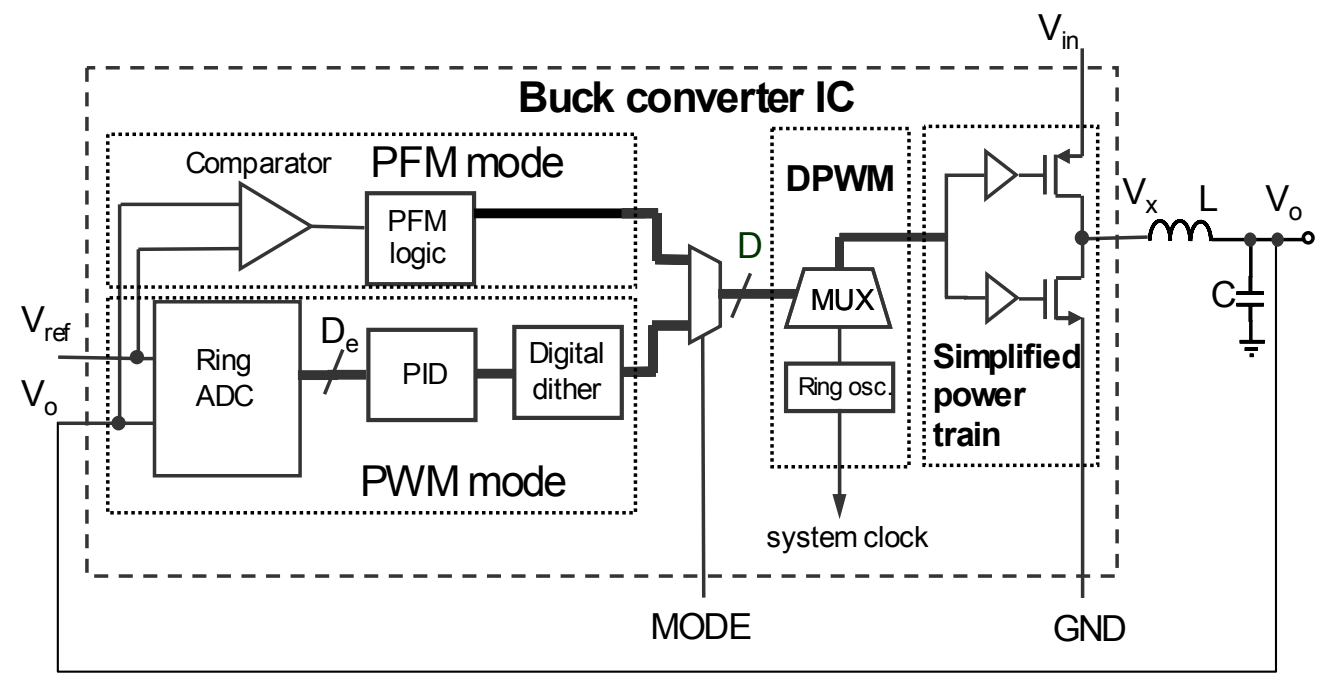

Fig. 1. Block diagram of dual-mode buck converter IC and external LC filter.

presented in Section III. The DPWM runs in both PWM and PFM modes, thus low power is a primary design objective for this block. A very low power DPWM scheme based on a ring oscillator-multiplexer structure (ring-MUX DPWM) is developed, the details of which are presented in section IV.

A prototype IC takes $2 \mathrm{~mm}^{2}$ active area in a $0.25-\mu \mathrm{m}$ CMOS process. With a cascode power train and internal power management, the IC demonstrates safe operation of a standard CMOS process rated for $2.75 \mathrm{~V}$ with a $5.5 \mathrm{~V}$ supply. The power train on the chip is rated for $400 \mathrm{~mA}$ load current, and efficiency above $90 \%$ is achieved for a load range of approximately 50-300 mA under PWM operation. Efficiency above $70 \%$ is achieved over the load range of 0.1 $400 \mathrm{~mA}$ in PFM mode. The latter result is enabled by a 4 $\mu \mathrm{A}$ quiescent current in PFM mode. Further experimental results are detailed in section $\mathrm{V}$.

\section{Architecture And Voltage Compatibility}

\section{A. PWM Mode}

The PWM mode runs the converter in continuous conduction. As shown in Fig. 1, in PWM mode, the error voltage $v_{e}=V_{o}-V_{\text {ref }}$ is quantized by the ADC to provide an error signal in the digital domain $D_{e}$, and the digital PID compensation network generates a duty ratio command $D$. The DPWM converts the duty ratio command into a PWM signal that controls the high side and the low side switches.

Subharmonic limit cycles may occur in a digitally controlled dc-dc converter because of the presence of two quantization operations, one in the $\mathrm{ADC}$ and one in the DPWM. Sufficient conditions to avoid subharmonic limit cycles are given in [1]. One step to avoid subharmonic limit cycles is the use a modulator (DPWM) that has higher resolution (smaller digital bin size) than that of the ADC. To meet this limit cycle avoidance condition, as well as the dc voltage precision specifications, the ADC quantization step size is designed to be $16 \mathrm{mV}$, and the DPWM step size is designed to be $5.4 \mathrm{mV}$. We note that this is one convenient design choice, but that substantially smaller step sizes can be readily implemented with the technology at hand.

A digital dither method that effectively reduces the DPWM hardware requirement, while keeping the same output resolution is employed [1]. With this method, 10-bit output resolution is achieved with a 5-bit hardware DPWM and 


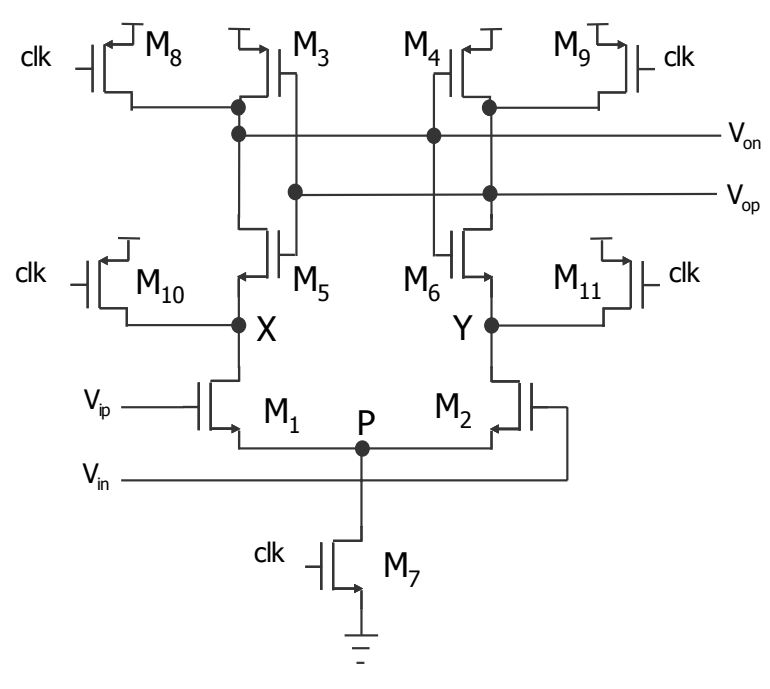

Fig. 2. Zero-bias-current comparator.

a 5-bit digital dither modulation process. The DPWM is implemented with a very low power ring oscillator-MUX structure reported in [2]. The ring oscillator in the DPWM runs at the switching frequency $f_{s}$, and provides clock signals for the whole system. Thus, high modulation resolution is obtained without any high frequency clock. The digital dither modulation provides very fine scale resolution of an additional five bits, by modulating the pattern for the hardware LSB over a 32 clock cycle period [1].

\section{B. PFM Mode}

The PFM mode runs the converter in discontinuous conduction mode with variable switching frequency and fixed on-time. Referring again to Fig. 1, a comparator samples the output voltage $V_{o}$ at a fixed frequency $f_{\text {samp, } P F M}$ and compares it to the reference $V_{\text {ref }}$. When $V_{o}<V_{\text {ref }}$, the controller generates a fixed-on-time pulse through the DPWM to charge up the output capacitor. Otherwise, the converter and the controller are idling. The switching frequency in PFM mode scales roughly proportionally to the load current, thus the switching loss is greatly reduced in light load conditions. Thus, PFM mode enables high efficiency with ultra-light loads, and is ideal to control the converter in standby mode.

The PFM mode quiescent power is the fundamental limitation on ultra-light load efficiency. To reduce it, a clocked zero-dc-current comparator [3] is used, as shown in Fig. 2. When the clock signal $c l k$ is high, the comparator is in reset mode. Nodes $V_{o p}, V_{o n}, X$ and $Y$ of the comparator are precharged to supply voltage by $M_{8}-M_{11}$, and node $\mathrm{P}$ is discharged to ground by $M_{7}$. When $c l k$ goes low, the comparator goes into evaluation and regeneration mode. Input pair $M_{1}$ and $M_{2}$ resolves the input voltage, and the differential current develops a voltage difference between nodes $X$ and $Y$ before the cross-coupled latch turns on. Then, the latch transistors $M_{3}-M_{6}$ regenerate the amplified output signal back to full swing. After the output signals are fully regenerated, the cross-coupled inverter pair holds the logic levels without taking any power. Very low power is consumed by this comparator since only dynamic current flows through the input pair and the regeneration pair during the evaluation transient, and once the output signals are fully established, there is no dc current flowing in the comparator.

\section{Voltage Compatibility and Internal Power Management}

Small feature size processes with intrinsic low supply voltage are preferred to implement the digital circuit to achieve small die area, high speed and low power. A 0.25$\mu \mathrm{m}$ CMOS process with highest allowable supply of $2.75 \mathrm{~V}$ is used to implement the IC. As mentioned in Section I, the battery in cellular phones supplies a voltage range between $5.5 \mathrm{~V}$ and $2.8 \mathrm{~V}$. Thus, the circuit cannot run directly from the battery, and internal power management is introduced to ensure voltage compatibility.

As shown in Fig. 3, a cascode structure is used to protect the power train switches from high input voltage. An intermediate voltage $V_{m}=V_{i n} / 2$ is needed to bias the cascode transistors $M_{P 2}$ and $M_{N 2}$, and to power the digital 


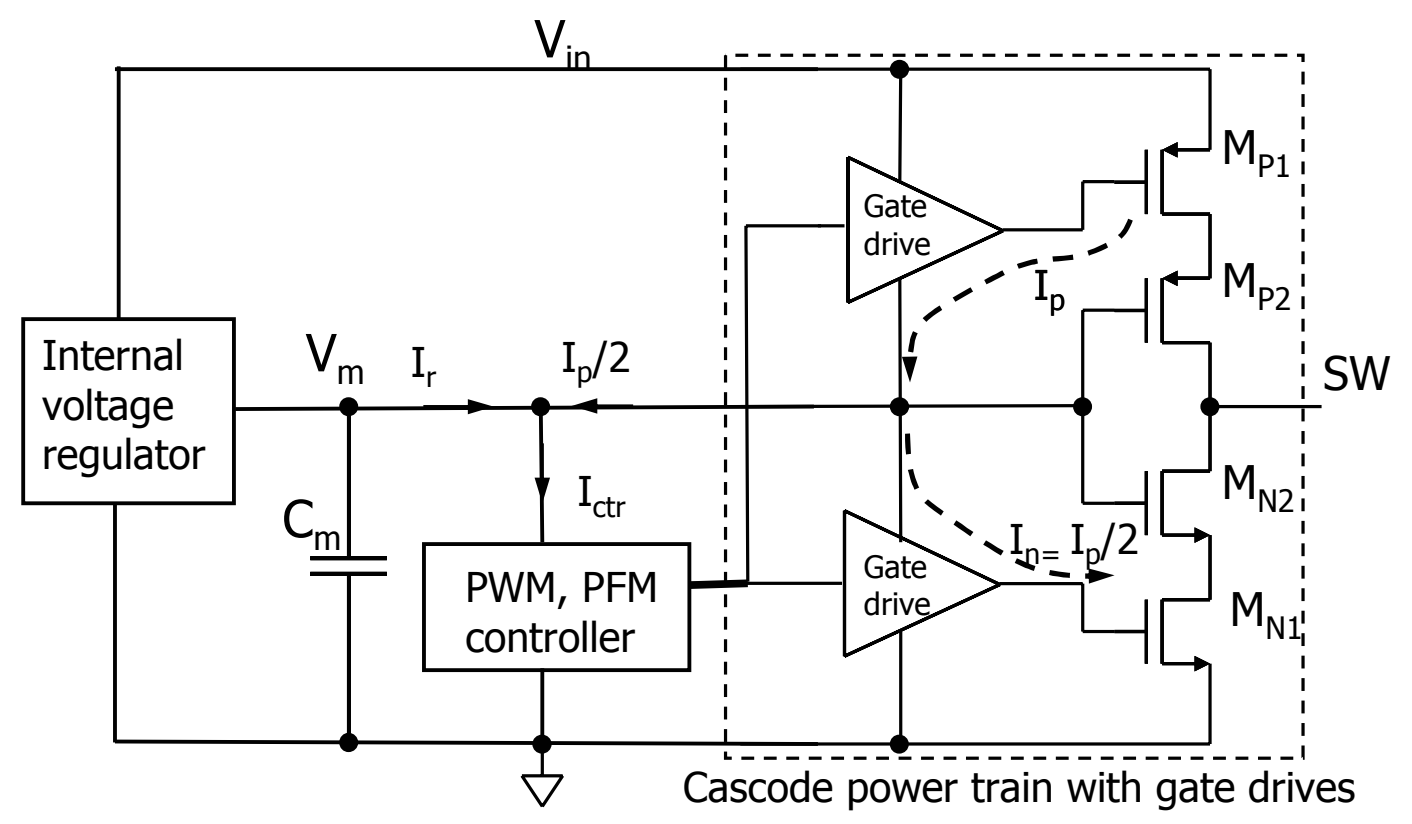

Fig. 3. Block diagram of internal power management.

circuits. A very low bias current internal class B regulator is used to provide a stable $V_{m}$.

The high side gate driver works between supplies $V_{\text {in }}$ and $V_{m}$, and the low side gate driver works between $V_{m}$ and ground. In each switching cycle, the average current $I_{p}$ flowing into node $V_{m}$ through the high side gate driver is approximately twice the current $I_{n}$ flowing out of $V_{m}$ through low side gate driver, since the power train PMOS transistor has twice the width of the NMOS transistor in this design to optimize conduction loss. The difference current $I_{p}-I_{n}$ can be used as a partial or complete supply for the controller. The total current consumed by the controller and the gate drivers can be calculated by summing the equivalent $\mathrm{dc}$ current that flows into the ground node. If the internal power management scheme were not employed, the resulting total current consumption would be $I_{p}+I_{n}+I_{c t r}$, where $I_{c t r}$ is the equivalent dc current drawn by the controller. With the internal power management scheme, the overall current consumption reduces to $I_{n}+I_{c t r}$. The dc bias current

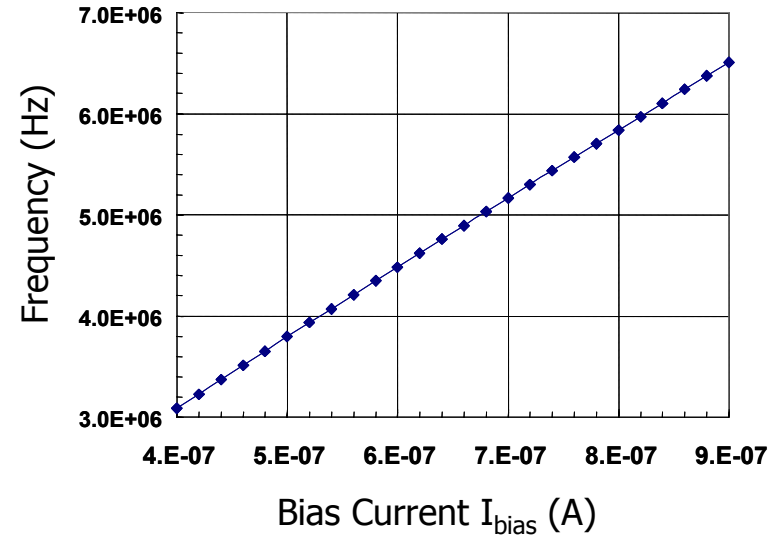

Fig. 4. Simulated frequency-current dependency of a 4-stage differential ring oscillator biased in subthreshold region.

in the class B internal regulator is more than two orders of magnitude lower than $I_{c t r}$ alone and is thus neglected. Therefore, a current savings of $I_{p}$ is achieved in PWM mode by using the internal power management scheme.

\section{RING-OSCILLATOR ADC}

In PWM mode, since $V_{o}$ is regulated to be in the vicinity of $V_{\text {ref }}$, an $\mathrm{ADC}$ that can handle a rail-to-rail input is 


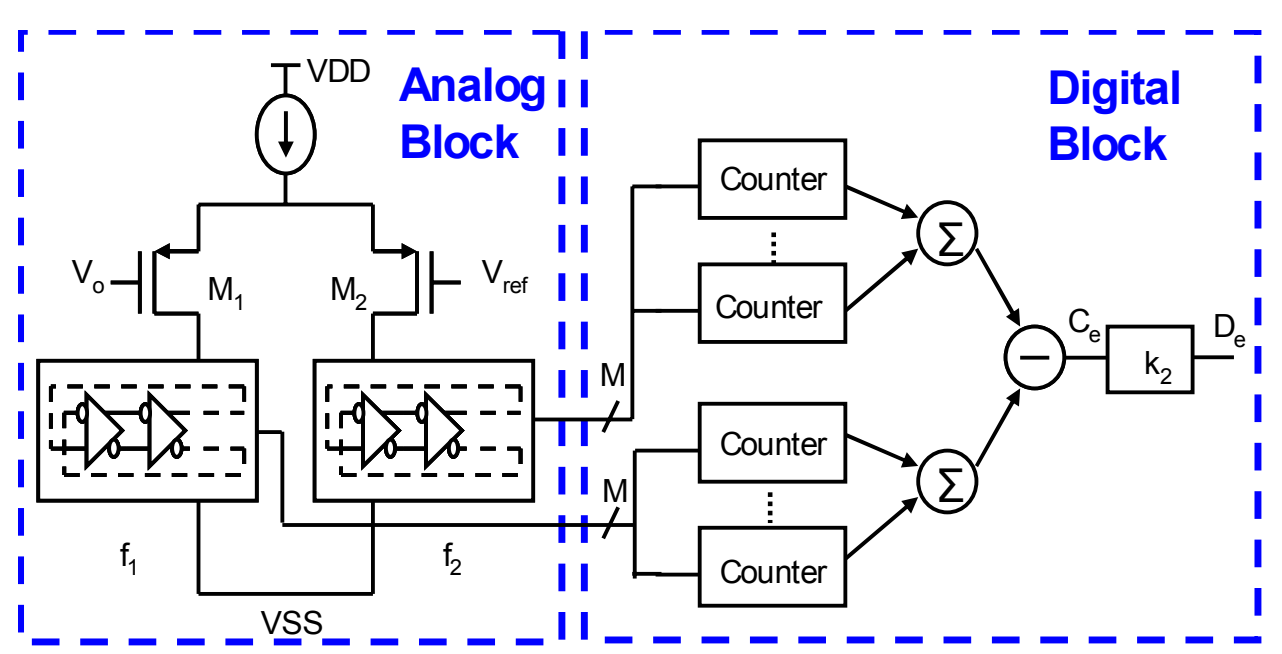

Fig. 5. Block diagram of ring-ADC.

not necessary, and a windowed ADC that realizes high resolution with reduced quantization range can be used [2], [4]. The main idea is to reduce the quantization window to the possible $V_{o}$ variation range, which is usually tens of millivolts centered at $V_{\text {ref }}$. Due to switching activities of the power train, disturbances might be observed on $V_{o}$. Therefore, an averaging ADC that is insensitive to switching noise and has a windowed quantization range is desirable.

Synthesizable ADC's based on VCO or delay-line structures have been reported [5], [6]. In this work, we develop an averaging windowed ring-ADC which is nearly entirely synthesizable. Compared to the VCO or delay-line based $\mathrm{ADC}$, the resolution of the developed ring-ADC is invariant under reference voltage changes, and can be flexibly adjusted to meet a wide range of applications.

The design of the ring-ADC is based on the observation that the oscillation frequency in a CMOS ring oscillator, biased in subthreshold region, has a linear dependency on the bias current, as illustrated in Fig. 4. The block diagram of the ring-ADC is shown in Fig. 5. The ADC consists of a simple analog block and a synthesizable digital block. A differential transistor pair $M_{1}-M_{2}$ drives two identical ring oscillators as a matched load. The bias current is such that the voltage swing on the ring oscillator is below threshold. Thus each ring oscillator's frequency is linearly dependent on its supply current. The error voltage $v_{e}$ develops differential current in the two branches that results in instantaneous differential frequency at the two oscillators. The frequency of each oscillator is captured by a counter that is reset at the beginning of each sampling cycle. At the end of the cycle, one counter output is subtracted from the other, and the result $C_{e}$ is used to calculate digitized error voltage $D_{e}$. Since there is uncertainty in the initial and ending phase, instead of looking at one output per ring, all the $\mathrm{M}$ taps on each ring oscillator are observed for frequency information. It can be shown that, ignoring quantization error and assuming good linearity in the input differential pair, $C_{e}$ is given by

$$
C_{e}=M k_{1} g_{m} T_{A D C} v_{e}
$$

where $M$ is the number of taps on each ring, $k_{1}$ is the ring oscillator frequency sensitivity function to bias current, $g_{m}$ is the transconductance of the input differential pair, and $T_{A D C}$ is the ADC sampling period, which equals the switching period $T_{s}$ of the converter. Finally, digitized error voltage 
$D_{e}$ is calculated by rounding off $C_{e}$. Since the saturation behavior of the input differential pair is dependent on the ratio of the input signal and the overdrive voltage of the differential pair, good linearity of the input transistors can be obtained by designing the overdrive voltage a few times higher than the differential input voltage. Since $v_{e}$ is usually less than $100 \mathrm{mV}$, the overdrive voltage needs to be only a few hundred millivolts.

In this design, a ring-ADC with $16 \mathrm{mV}$ quantization bin size, and a total quantization window of $80 \mathrm{mV}$ takes $0.15 \mathrm{~mm}^{2}$ chip area. At $500 \mathrm{kHz}$ sampling frequency, the measured current consumption of this ADC is $37 \mu \mathrm{A}$. The digital block can be further simplified by using a decoder and a counter, instead of a number of counters, to monitor the signals on all the taps in each ring, resulting in significantly smaller die area and much lower power consumption. Compared to ADC's based on a VCO or a delay-line, this ring-ADC has invariant resolution under different reference voltage levels due to the common mode rejection capability of the differential pair, thus is suitable for a wide range of applications. Furthermore, the resolution of the ring-ADC can be controlled through the bias current, which can be made either constant or adjusted for automatic gain control. For example, the bias current in the differential pair of the ring-ADC can be made a function inverse to the square of the input voltage. Thus when the input voltage reduces, the gain of the ADC increases, and hence the controller gain is also raised proportionally, resulting in a stabilized loop gain. In summary, the ring-ADC has low power and small area, and its resolution can be designed with high flexibility depending on application requirements. The quantization resolution of the ring-ADC can be scaled by changing the number of stages in the ring, and varying the bias current of the differential pair.

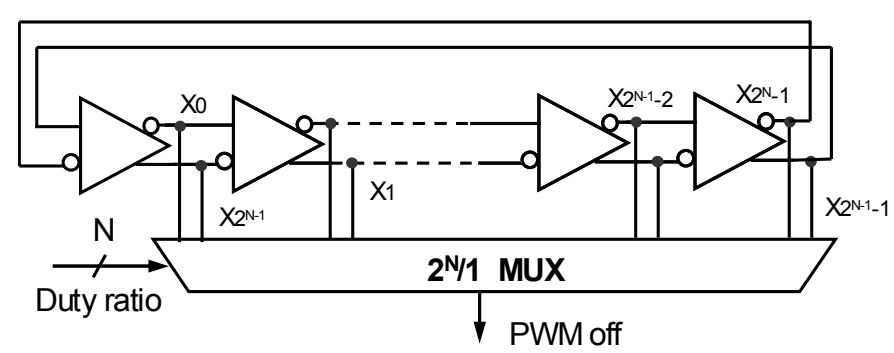

(a)

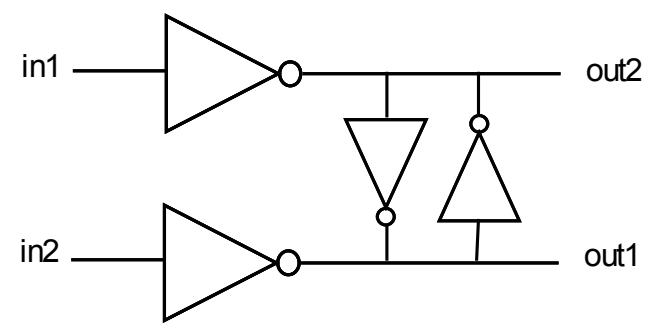

(b)

Fig. 6. (a) Block diagram of an N-bit ring-MUX DPWM, (b) the delay stage of the differential ring oscillator in the DPWM.

\section{RING-MUX DPWM}

One method to digitally create PWM signals is with a fastclocked counter-comparator scheme [7]. Such a design takes reasonable die area but the power consumption reported is on the order of mW's because of the high clock frequency in the counters [8]. A tapped delay line scheme is proposed in [9]. Power is significantly reduced with respect to the fastcounter-comparator scheme since the fast clock is replaced by a delay line which runs at the switching frequency of the converter. A combined delay-line-counter structure is reported in [5], [10], aiming to make a compromise between area and power.

A ring-oscillator-MUX implementation of a DPWM module, with block diagram shown in Fig. 6(a), has area and power considerations similar to those of the delay line approach. The ring oscillator runs with subthreshold voltage 


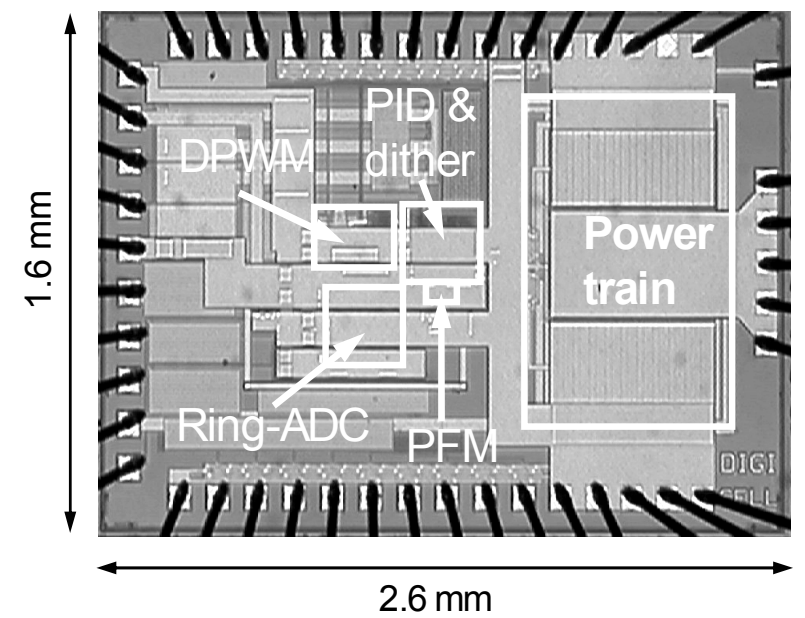

Fig. 7. Chip micrograph.

swing, thus the power consumption is much lower than the delay-line structure due to the reduced swing. Since the ring oscillator in the DPWM runs in current starved mode, the frequency can be controlled by adjusting the supply current to the entire ring. Thus the switching frequency of the converter can be locked to an external clock by controlling the DPWM ring current. The ring oscillator in the DPWM also generates the clock signals for the entire converter system.

The main components of the ring-MUX scheme are an $\mathrm{N}$-stage differential ring oscillator, which yields $2 \mathrm{~N}$ symmetrically oriented taps, and a 2N/1 MUX that can select appropriate signals from the ring. The delay stage in the differential ring oscillators in the DPWM is shown in Fig. 6(b). A square wave propagates along the ring. When the rising edge reaches tap $X_{0}$ in the ring, the rising edge of the PWM signal is generated. The falling edge of this PWM signal is generated when the rising edge of the propagating square wave reaches a specified tap in the ring. The MUX is used to specify the tap in accord with the commanded duty cycle.

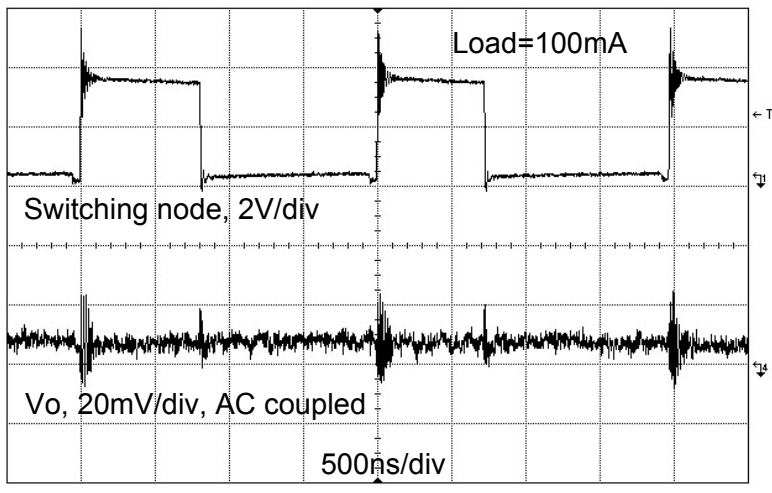

Fig. 9. Experimental steady-state response in PWM mode with $V_{i n}=3.2$ $\mathrm{V}, V_{o}=1.2 \mathrm{~V}$, and $f_{s}=500 \mathrm{kHz}$.

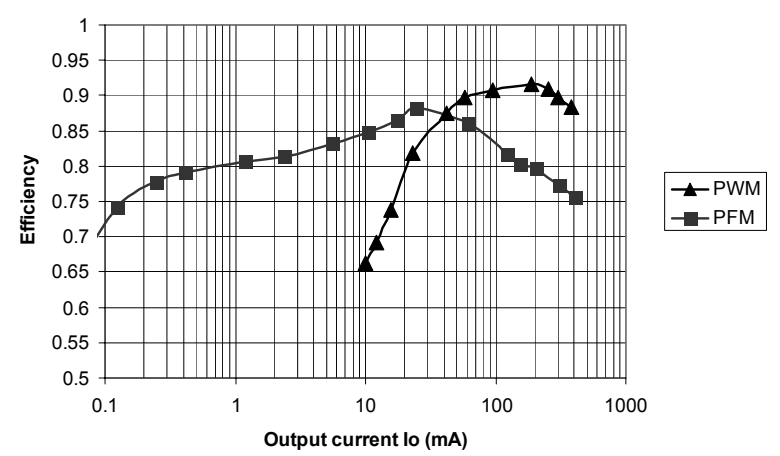

Fig. 10. Measured PWM and PFM mode converter efficiency as functions of output current, with $V_{i n}=4 \mathrm{~V}$ and $V_{o}=1.5 \mathrm{~V}$.

\section{EXPERIMENTAL RESULTS}

The complete dual-mode digitally-controlled buck converter IC is implemented in a $0.25-\mu \mathrm{m}$ CMOS process. The die photo of the chip is shown in Fig. 7. The total chip area is $4 \mathrm{~mm}^{2}$, out of which $2 \mathrm{~mm}^{2}$ is the active area. The required pin count for the buck converter IC is 10 , and all the other pins are for test purposes. Table I summarizes the application and the measured performance of the IC.

Experimental closed-loop load transient responses with a current load step of $100 \mathrm{~mA}$ for PWM mode and PFM mode, respectively, are shown in Fig. 8. In PWM mode, it can be seen that the dc voltages at high load and low load are within the zero error bin of the ADC, which is $16 \mathrm{mV}$. In PFM 


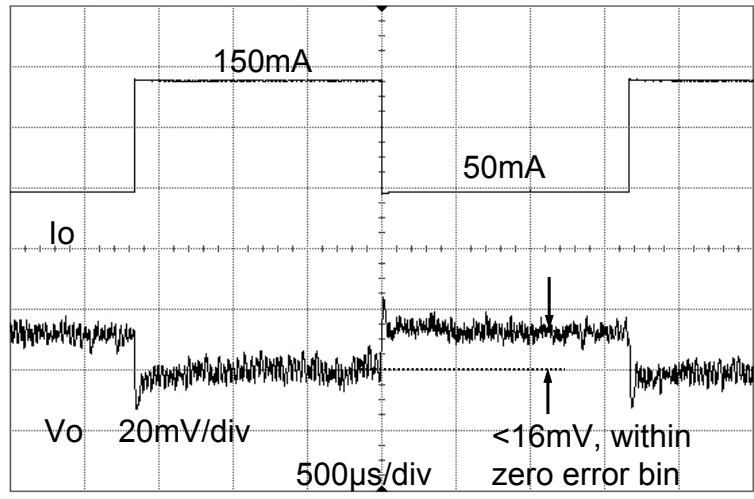

(a)

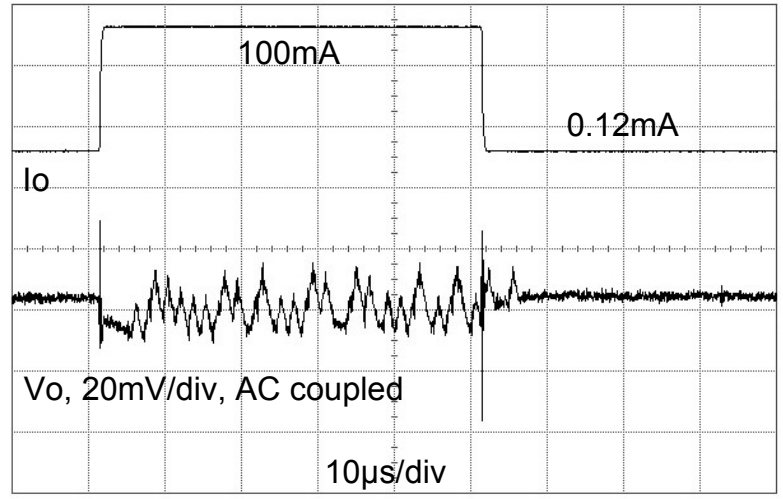

(b)

Fig. 8. Experimental load transient response with $V_{i n}=3.2 \mathrm{~V}, V_{o}=1.2 \mathrm{~V}$ (a) PWM mode response with $f_{s}=1 \mathrm{MHz}$, (b) PFM mode response with $f_{\text {samp }, P F M}=600 \mathrm{kHz}$.

TABLE I

CHIP PERFORMANCE SUMMARY.

\begin{tabular}{|l|l|}
\hline Technology & $0.25-\mu \mathrm{m}$ CMOS (Max. supply 2.75 V) \\
\hline Input voltage range & $5.5-2.8 \mathrm{~V}$ \\
\hline Output voltage range & $1.0-1.8 \mathrm{~V}$ \\
\hline External LC filter & $\mathrm{L}=10 \mu \mathrm{H}, \mathrm{C}=47 \mu \mathrm{F}$ \\
\hline Maximum load current & $400 \mathrm{~mA}$ \\
\hline PFM mode sampling frequency & $600 \mathrm{kHz}$ \\
\hline PFM mode quiescent current & $4 \mu \mathrm{A}$ \\
\hline PWM mode switching frequency & $0.5-1.5 \mathrm{MHz}$ \\
\hline PWM mode dc output voltage precision & $\pm 0.8 \%$ \\
\hline PWM mode output voltage ripple & $2 \mathrm{mV}$ \\
\hline Active chip area & $2 \mathrm{~mm}^{2}$ \\
\hline
\end{tabular}

mode, the voltage excursion is below $30 \mathrm{mV}$ when the load current is $100 \mathrm{~mA}$. Steady-state waveforms in PWM mode are shown in Fig. 9. Efficiencies as a function of load current of the buck converter in both PWM mode and PFM mode are measured with $V_{i n}=4 \mathrm{~V}$ and $V_{o}=1.5 \mathrm{~V}$, as illustrated in Fig. 10. An efficiency of $92 \%$ is achieved in PWM mode with load current of $189 \mathrm{~mA}$. It can be observed that $40 \mathrm{~mA}$ is the crossover point of the PWM and the PFM efficiency curves. Thus, when load current is lower than $40 \mathrm{~mA}$, the converter should be switched from PWM to PFM mode for better efficiency. Furthermore, over a $20 \mathrm{~mA}$ to $200 \mathrm{~mA}$ load current range, high efficiency (over 80\%) can be observed in each mode, thus it is easy to implement a scheme to switch between the two modes due to the wide load range of overlapping high efficiency. 


\section{CONCLUSION}

This paper describes an ultra-low-quiescent-current dualmode digital controller IC for high frequency dc-dc buck converters. Internal power management and power switch structures enable the use of a small feature size $(0.25-\mu \mathrm{m})$ CMOS process with voltages up to $5.5 \mathrm{~V}$. The paper further demonstrates sub-threshold operation of CMOS transistors as a viable, very low power option for analog-digital interface elements. The work illustrates the promise of implementing digital power management IC's in standard CMOS processes, with low power and small area, using a combination of digital processing and special purpose analog-digital interface structures.

\section{ACKNOWLEDGMENT}

The authors greatly appreciate the guidance of Prof. Y. C. Liang of the National University of Singapore in the power train design during his visit from 2001 to 2002. We also thank National Semiconductor for the IC fabrication and Joe Emlano of National Semiconductor for chip packaging.

\section{REFERENCES}

[1] A. V. Peterchev and S. R. Sanders, "Quantization resolution and limit cycling in digitally controlled PWM converters," in IEEE Transactions on Power Electronics, vol. 18, no. 1, Jan 2003.

[2] J. Xiao, A. V. Peterchev, and S. R. Sanders, "Architecture and IC implementation of a digital VRM controller," in Proc. IEEE Power Electron. Spec. Conf., vol. 1, 2001, pp. 38-47.

[3] Y.-T. Wang and R. B., "An 8-bit 150-MHz CMOS A/D converter," in IEEE Journal of Solid-State Circuits, vol. 35, no. 3, March 2000.

[4] A. V. Peterchev, J. Xiao, and S. R. Sanders, "Architecture and IC implementation of a digital VRM controller," in IEEE Transactions on Power Electronics, vol. 18, no. 1, Jan 2003.

[5] G.-Y. Wei, J. Kim, D. Liu, S. Sidiropoulos, and M. Horowitz, "A variable-frequency parallel $\mathrm{I} / \mathrm{O}$ interface with adaptive power-supply regulation," IEEE JSSC, vol. 35, no. 11, pp. 1600-1610, Nov. 2000.

[6] B. Patella., A. Prodic, A. Zirger, and D. Maksimovic, "High-frequency digital PWM controller IC for DC-DC converters," in IEEE Transactions on Power Electronics, vol. 18, no. 1, Jan 2003.

[7] G.-Y. Wei and M. Horowitz, "A low power switching power supply for self-clocked systems," in IEEE Int. Symp. Low Power Electronics and Design Dig. Tech. Papers, Aug. 1996, pp. 313-317.

[8] —, "A fully digital, energy-efficient, adaptive power-supply regulator," IEEE JSSC, vol. 34, no. 4, pp. 520-528, apr 1999.

[9] A. P. Dancy and A. P. Chandrakasan, "Ultra low power control circuits for PWM converters," in Proc. IEEE Power Electron. Spec. Conf., vol. 1, 1997, pp. 21-27.

[10] A. P. Dancy, R. Amirtharajah, and A. P. Chandrakasan, "Highefficiency multiple-output DC-DC conversion for low-voltage systems," IEEE Transactions on VLSI Systems, vol. 8, no. 3, pp. 252-263, June 2000 